\title{
Evaluación del papel del licopeno en la tolerancia a los daños por frío durante la conservación refrigerada de frutos de pomelo rojo
}

\section{Evaluation of the role of lycopene in the tolerance to chilling injury during cold storage of red grapefruit}

Lado, Joanna (1), Rodrigo, María Jesús (2), Zacarías, Lorenzo (2)

(1) Instituto Nacional de Investigación Agropecuaria (INIA), Salto, Uruguay -

(2) Instituto de Agroquímica y Tecnología de Alimentos (IATA), Consejo Superior de Investigaciones Científicas (CSIC), Valencia, España.

Contacto: jlado@inia.org.uy

RECIBIDO: 16/2/2016 - APROBADO: 1/7/2016

\begin{abstract}
Resumen
Los frutos de pomelo son sensibles a desarrollar lesiones en la piel, conocidas como daño por frío (DF), durante la conservación a bajas temperaturas. En el pomelo rojo Star Ruby los DF se restringen a las zonas amarillas de la piel, mientras que las zonas rojas, que acumulan el caroteno licopeno, permanecen intactas. El DF está asociado a la producción de especies reactivas del oxígeno y el licopeno presenta gran capacidad antioxidante, por lo que este pigmento podría estar implicado en la tolerancia frente al daño oxidativo. Para comprobar esta hipótesis se comparó el comportamiento a baja temperatura $\left(2^{\circ} \mathrm{C}\right)$ entre frutos de pomelo con diferencias en el contenido de licopeno $(0,92 \mathrm{vs} .45,2 \mu \mathrm{g} / \mathrm{gPF})$. Los frutos con alta concentración de licopeno (50 veces superior) fueron más tolerantes al DF y tuvieron una mayor capacidad (2-3 veces) para secuestrar el oxígeno singlete (SOAC). El estudio del sistema antioxidante enzimático y no enzimático (ácido ascórbico y glutatión) no reveló un papel relevante en la protección frente al DF. Los resultados sugieren que el oxígeno singlete estaría implicado en el daño oxidativo provocado por el frío en los frutos de pomelo y que el licopeno protegería del daño celular producido por esta especie reactiva, incrementado la tolerancia.
\end{abstract}

Palabras clave: Almacenamiento refrigerado, citrus, carotenoides, licopeno, poscosecha.

\begin{abstract}
Grapefruit are sensitive to develop chilling injury (CI) symptoms during low temperature storage. In red grapefruit Star Ruby chilling damage during cold storage was restricted to yellow areas of the skin, while the red areas that accumulate lycopene remain largely unaffected. CI has been associated with the production of reactive oxygen species and lycopene has potent antioxidant capacity, thus this pigment might be responsible or be involved in the tolerance against oxidative damage. To test this hypothesis, we have compared the postharvest behavior at low temperatures between grapefruit with great differences in lycopene content $(0,92 \mathrm{vs} .45,2 \mu \mathrm{g} / \mathrm{gPF})$. Fruit with high concentration of lycopene in the skin (50 times) were more tolerant to CI and at the same time had a higher capacity (2-3 times) to scavenger singlet oxygen radical (SOAC). The study of the enzymatic antioxidant and non-enzymatic (ascorbic acid and glutathione) systems did not revealed an important role in the protection against CI. Collectively, these results suggest that in grapefruits singlet oxygen would be involved in the oxidative damage caused by storage at low temperatures and that lycopene may protect against the cell damage caused by this reactive oxygen species, increasing then the tolerance to cold stress.

Keywords: Carotenoids, citrus, cold storage, lycopene, postharvest.
\end{abstract}

\section{Introducción}

Los frutos de pomelo se encuentran entre los cítricos más sensibles a desarrollar daños por frío (DF) durante la con- servación a temperaturas inferiores a $10^{\circ} \mathrm{C}$. Estos daños se manifiestan como picados, hendiduras y ennegrecimiento en la piel, que deprecian considerablemente la calidad comercial de los frutos (Lafuente y Zacarías, 2006). En trabajos previos 
se ha comprobado que los frutos de pomelo de la variedad Star Ruby, que acumulan el caroteno licopeno en la piel y la pulpa $y$, por lo tanto, tienen coloración roja, desarrollan severos daños en la piel cuando se almacenan a $2^{\circ} \mathrm{C}$, especialmente en las zonas con tonalidades verdes o amarillas, mientras que las zonas rojizas o más coloreadas permanecen prácticamente intactas (Lado, et al., 2015a). Este efecto podría estar relacionado con la capacidad antioxidante del licopeno, ya que existen numerosas evidencias que indican la implicación del estrés oxidativo en la inducción y desarrollo de los daños por frío en los frutos cítricos (Sala y Lafuente, 2000). El licopeno es un carotenoide lineal que posee un total de 11 dobles enlaces en la molécula, lo que le confiere una significativa actividad antioxidante (Singh y Goyal, 2008), con el doble de capacidad para secuestrar el radical ${ }^{1} \mathrm{O}_{2}$ que el $\beta$-caroteno y 10 veces respecto al $\alpha$-tocoferol (Di Mascio, et al., 1989).

Durante años se han acumulado numerosos resultados en distintas especies que demuestran la activación de los diferentes procesos que conforman el sistema antioxidante en los vegetales como respuesta al estrés por bajas temperaturas (Toivonen, 2004). En los frutos cítricos se ha comprobado que la actividad de la enzima catalasa tiene un papel determinante en la protección frente a los DF (Sala y Lafuente, 2000). A su vez, se ha demostrado también que algunos frutos con mayor concentración de pigmentos (carotenoides o antocianos) son capaces de resistir más eficazmente a distintos tipos de estrés y reducir el ataque de patógenos. Tal es el caso de tomate con una elevada acumulación de antocianos (Zhang, et al., 2013), lo que conlleva un aumento de su vida postcosecha. Resultados similares se han descrito en boniato, donde un aumento del contenido de $\beta$-caroteno en plantas transgénicas provocó una mayor actividad antioxidante total y un aumento en la resistencia a estrés salino (Kim, et al., 2012). En este sentido, la tolerancia de las zonas rojas del pomelo rojo Star Ruby frente al DF podría estar relacionada también con una mayor activación del sistema antioxidante en las zonas rojas de los frutos que acumulan licopeno, frente a las zonas amarillas de estos frutos o de frutos amarillos de otras variedades de pomelo.

Con el objetivo de comprobar esta hipótesis y aprovechando que los frutos de pomelo rojo Star Ruby acumulan elevadas cantidades de carotenoides totales, y especialmente licopeno, cuando se desarrollan en condiciones de oscuridad (Lado, et al., 2015b), se evaluó la incidencia de los DF durante 8 semanas de conservación a $2{ }^{\circ} \mathrm{C}$ en frutos de coloración amarilla normal (frutos control) frente a frutos de piel de color rojo intenso desarrollados en oscuridad. Paralelamente, se analizaron las actividades del sistema antioxidante enzimático (enzimas catalasa-CAT, superóxido dismutasa-SOD, ascorbato peroxidasa-APX y glutatión reductasa-GR) y no enzimático (ácido ascórbico y glutatión) y se evaluó la capacidad del tejido para inactivar especies reactivas del oxígeno, especialmente oxígeno singlete, durante el almacenamiento refrigerado.

\section{Materiales y Métodos}

\section{Material vegetal y diseño experimental}

Se cosecharon frutos maduros de pomelo rojo (Citrus paradisi) de la variedad Star Ruby en el Instituto Valenciano de Investigaciones Agrarias (IVIA) en Moncada, Valencia (España), en diciembre de 2013. Para estimular la acumulación de licopeno y, por tanto, la coloración roja de la piel, un lote de 50 frutos, aproximadamente a mitad del número final, se taparon con plástico negro entre finales de julio y principios de agosto, evitando la exposición a la luz hasta el momento de la cosecha (SR-rojo). Como frutos control (SR-control) se utilizaron frutos localizados en el exterior de los mismos árboles y expuestos directamente a la luz, por lo que presentaron la tonalidad amarilla característica (Figura 1). Los detalles del experimento se describen en Lado et al. (2015b). Los frutos de SR-control (no tapados) y SR-rojo (tapados) fueron cosechados en madurez fisiológica y se almacenaron durante 8 semanas a $2{ }^{\circ} \mathrm{C}$.

El diseño experimental fue completamente al azar con tres repeticiones por tratamiento y momento. Cada repetición estaba compuesta por 10 frutos (en el caso de la evaluación de DF y color). El resto de los frutos sometidos al tratamiento fueron utilizados para la obtención de tejido para el análisis de carotenoides. Para detectar posibles diferencias entre frutos control y frutos rojos se realizó una prueba $\mathrm{T}$ de comparación de medias, admitiendo una probabilidad de error de 0,05. En todos los casos se utilizó el software estadístico Infostat.

\section{Evaluación del daño por frío}

A lo largo del período de almacenamiento se determinó el daño por frío (DF) en la piel de los frutos SR-control y SR-rojo mediante la incidencia (número de frutos con síntomas/ número total de frutos * 100) y el índice de daño por frío (IDF) a las 2, 4 y 8 semanas de almacenamiento, para estimar la severidad de los daños. Para ello se utilizaron tres repeticiones de 10 frutos por tratamiento, los cuales se clasificaron en diferentes categorías utilizando una escala visual, según el siguiente criterio: $0=\sin$ daños visibles; $1=$ daños ligeros, abarcando hasta un $10 \%$ de la superficie del fruto; $2=$ daños moderados, abarcando entre 10 y $50 \%$ de la superficie del fruto; $3=$ daños severos, abarcando más del $50 \%$ de la superficie del fruto (Lafuente, et al., 1997). El IDF se calculó mediante la fórmula: $\sum$ (número de frutos de cada categoría $\mathrm{x}$ valor de cada categoría)/ número total de frutos examinados.

\section{Determinación del color de la piel}

Para la determinación del color se utilizó un colorímetro Minolta CR-330, registrándose las coordenadas Hunter $L^{*} a^{\star} y$ $b^{\star}$ que se representaron como la relación $\mathrm{a} / \mathrm{b}$. Un valor de $\mathrm{a} / \mathrm{b}$ negativo se refiere a frutos de coloración verdosa, en torno a cero es el viraje de color y valores positivos indican coloración naranja-rojiza. Los mismos frutos que se utilizaron para la determinación del color se utilizaron para la obtención de tejido, pelando la parte externa y coloreada de la corteza (flavedo), que se congeló en nitrógeno líquido, se trituró en un molinillo e inmediatamente se almacenó a $-80^{\circ} \mathrm{C}$ para posterior análisis.

\section{Extracción, cuantificación e identificación de carotenoides}

La extracción y cuantificación de carotenoides se llevó a cabo a partir de $0,5 \mathrm{~g}$ de tejido de flavedo según protocolo detallado descrito por Rodrigo et al. (2003). Al finalizar la extracción, la muestra se llevó a sequedad completa mediante corriente de $\mathrm{N}_{2}$, se resuspendió en $1 \mathrm{ml}$ de acetona y se incubó durante la noche a $-20^{\circ} \mathrm{C}$. Tras centrifugar a $13000 \mathrm{~g}$ durante $10 \mathrm{~min}$ a $4{ }^{\circ} \mathrm{C}$, el sobrenadante se transfirió a un nuevo vial, se llevó a sequedad en corriente de $\mathrm{N}_{2}$ y se almacenó en atmósfera 
de $\mathrm{N}_{2} \mathrm{a}-20{ }^{\circ} \mathrm{C}$ hasta el momento de análisis. Durante todo el procedimiento se tuvo especial atención en evitar la exposición a la luz de las muestras trabajando en condiciones de baja luminosidad, con el fin de evitar la fotodegradación, la isomerización y los posibles cambios estructurales de los carotenoides. Todas las muestras se extrajeron al menos por triplicado. La identificación y cuantificación de los carotenoides individuales se realizó por cromatografía líquida de alta resolución acoplada a detector de fotodiodos (HPLC-PDA), según lo detallado en Alquezar et al. (2008).

\section{Determinación de actividad enzimática}

La extracción de proteínas se realizó a partir de $0,5 \mathrm{~g}$ de tejido congelado, utilizando un mortero en un baño con hielo a $4{ }^{\circ} \mathrm{C}$ y arena de mar como abrasivo. Se agregaron $2,5 \mathrm{ml}$ de tampón de extracción (fosfato de potasio $100 \mathrm{mM}$ y Triton X-100 $0,1 \%, \mathrm{pH} 7,5)$ y se lavó el mortero con $2,5 \mathrm{ml}$, recuperando el extracto en un tubo, previo filtrado a través de dos capas de muselina. El extracto se utilizó para la determinación de las cuatro actividades enzimáticas por espectrofotometría (Thermo-Spectronic Genesis UV10, USA), utilizando los siguientes tampones durante el proceso: para APX (EC1.11.1.11) se utilizó tampón PBS $50 \mathrm{mM}(\mathrm{pH} 7,1)$ suplementado con ascorbato de sodio $1 \mathrm{mM}$, EDTA $0,1 \mathrm{mM}$ y dos gotas de Triton X-100; para la actividad SOD (EC1.15.1.1), PBS 50mM 50mM (pH $7,8)$ con ácido dietil-diamino-pentacético 1,33 mM; para la actividad CAT (EC1.11.1.6) y GR (EC1.6.4.2) se utilizó tampón PBS pH 6,8 y 7,5, respectivamente.

La actividad APX se determinó siguiendo la reducción de la absorbancia a $290 \mathrm{~nm}$ provocada por el consumo de ácido ascórbico presente. La actividad SOD se determinó siguiendo la reducción del tetrazolio nitroblue inducida por peróxido de hidrógeno, utilizando el sistema xantina-xantina oxidasa. La actividad CAT se evaluó siguiendo la cinética de la reducción del cloruro de titanio frente al peróxido de hidrógeno y la actividad GR se analizó determinado durante 2 min el aumento de la absorbancia (412 nm) generado por el aducto DTNB-GSH al reducirse el glutatión oxidado (GSSG). Todos los ensayos se desarrollaron a $25^{\circ} \mathrm{C}$. El contenido de proteína soluble total se determinó utilizando BSA como estándar según el método de Bradford (1976). Todas las actividades se expresaron como unidades arbitrarias por mg de proteína. Los procedimientos/cinéticas realizados para cada enzima se detallan en Arbona et al. (2003).

\section{Determinación de contenido de ácido ascórbico (AsA) y dehidroascórbico (DHA)}

La extracción se realizó a partir de 0,5 g de tejido congelado con $4 \mathrm{ml}$ de ácido meta fosfórico $0,1 \%$. Los detalles de la extracción de AsA y DHA se especifican en Alós et al. (2014). La cuantificación de AsA se realizó mediante un HPLC (Dionex, Barcelona, España), equipado con un detector de fotodiodos y el software Chromeleon (Dionex, Barcelona, España). Una alícuota de $10 \mu \mathrm{L}$ de la muestra final se inyectó en el sistema cromatográfico equipado con una columna Ultrabase C18 $(100 \mathrm{~mm} 4,6 \mathrm{~mm} \times 2,5 \mu \mathrm{m})$ a $35^{\circ} \mathrm{C}$. La fase móvil consistió en metanol:agua $\mathrm{pH} 2,5(15: 85, \mathrm{v} / \mathrm{v})$ con un flujo de $0,2 \mathrm{ml} / \mathrm{min}$. La cuantificación de AsA se realizó a $248 \mathrm{~nm}$ utilizando un detector de fotodiodos. Para la cuantificación se utilizó una curva patrón realizada en un rango de concentraciones entre 1 y $100 \mu \mathrm{g} / \mathrm{ml}$, a partir de una solución del estándar de AsA disuelto en ácido metafosfórico 2\%. El contenido de DHA se calculó como la diferencia entre vitamina $C$ total y el contenido de AsA. La vitamina $C$ total se calculó como la suma de AsA + DHA. El contenido de DHA fue mínimo en relación al ácido ascórbico $(<8,0 \mathrm{mg} / 100 \mathrm{gPF})$ en todas las muestras, por lo cual no se presentan estos resultados. Todas las muestras se realizaron por triplicado.

\section{Determinación de glutatión}

La extracción se realizó a partir de $0,5 \mathrm{~g}$ de flavedo en mortero, utilizando $5 \mathrm{ml}$ de ácido metafosfórico al $5 \%$ a $4{ }^{\circ} \mathrm{C}$. A continuación se filtró la muestra con muselina y se centrifugó a $10000 \mathrm{~g}$ durante $15 \mathrm{~min}$ a $4{ }^{\circ} \mathrm{C}$. Una alícuota de $400 \mu \mathrm{L}$ se mezcló con $600 \mu \mathrm{L}$ de tampón fosfato $0,5 \mathrm{M}$ (pH 7,5). Se preparó la mezcla de reacción (RM) con NADPH 0,3 mM, DTNA $6 \mathrm{mM}$ y agua destilada y se atemperó durante 1 hora a $25^{\circ} \mathrm{C}$. Para la determinación del glutatión total, tanto la forma oxidada como la reducida (GSH+GSSG), se colocaron $50 \mu \mathrm{L}$ de agua destilada en $1 \mathrm{ml}$ de muestra diluida y se incubó a temperatura ambiente durante 1 hora (a). Para determinar el GSSG (glutatión oxidado), a $1 \mathrm{ml}$ de muestra diluida se agregaron $20 \mu \mathrm{L}$ de 2 -vinilpiridina y $30 \mu \mathrm{L}$ de trietanolamina y se incubó durante 1 hora a $25^{\circ} \mathrm{C}$ (b). Para la determinación en cada caso se colocaron $500 \mu \mathrm{L}$ de RM en una cubeta y se añadieron 200 $\mu \mathrm{L}$ de la muestra a o b. A continuación se añadieron $136 \mu \mathrm{L}$ de glutatión reductasa (GR), mezclando activamente para iniciar inmediatamente la lectura en espectrofotómetro a $412 \mathrm{~nm}$ durante 2 minutos. Se prepararon curvas estándar tanto para el GSH, a partir de una solución $100 \mathrm{mM}$, como para el GSSG, a partir de una solución $50 \mathrm{mM}$. Una vez obtenidos ambos valores, se calculó el glutatión reducido como el glutatión total (GSH+GSSG) - glutatión oxidado (GSSG). Los resultados se expresaron en $\mathrm{nmol} / \mathrm{g}$ de peso fresco.

\section{Determinación de actividad SOAC}

El procedimiento detallado para la determinación de la actividad SOAC se describe en Ouchi et al. (2010) y Aizawa et al. (2011), si bien se realizaron algunas modificaciones. Se realizó una extracción en un mortero, previamente refrigerado a $4{ }^{\circ} \mathrm{C}$, con $6 \mathrm{ml}$ de etanol/cloroformo/agua (50:50:1, v/v/v), utilizando arena de mar como abrasivo. El extracto fue filtrado con una capa de muselina y se utilizó una alícuota de $200 \mu \mathrm{L}$ en la reacción. La alícuota se mezcló con $2 \mathrm{ml}$ de 2,5-diphenil3,4-benzofurano 0,10 mM (DPBF, Sigma-Aldrich, Barcelona, España) y se agregó posteriormente $1 \mathrm{ml}$ de endoperóxido 1,5 mM (EP, Invitrotech, Japón). El DPBF se utilizó como la sonda indicadora de la capacidad para secuestrar el oxígeno singlete y el EP como generador de esta especie reactiva. Se registraron los cambios en la absorbancia del DPBF a $413 \mathrm{~nm}$ durante 1 hora a $35^{\circ} \mathrm{C}$ y se utilizó $\alpha$-tocoferol (Sigma-Aldrich, Barcelona, España) como estándar. El valor relativo SOAC para cada muestra se calculó como:

$\left(t_{1 / 2 \text { muestra }}-t_{1 / 2 \text { blanco }}\right) /\left(t_{1 / 2 \alpha \text { Toc }}-t_{1 / 2 \text { blank } \alpha \text { Toc }}\right)^{*}$ $([\alpha-\operatorname{Toc} \mathrm{g} / \mathrm{L}] /[$ muestra $\mathrm{g} / \mathrm{L}])$

donde: vida media de la muestra $\left(\mathrm{t}_{1 / 2 \mathrm{muestra}}\right)$; vida media del blanco $\left(t_{1 / 2 b l a n c o}\right)$; vida media del $\alpha$-Tocoferol $\left(t_{1 / 2 a \mathrm{Toc}}\right)$ y vida media del blanco utilizado con el $\alpha$-Tocoferol ( $\left.t_{1 / 2 b l a n k ~ a T o c}\right)$. 


\section{Resultados}

\section{Color, carotenoides e incidencia del DF}

Los frutos de pomelo Star Ruby cultivados en oscuridad durante las últimas etapas del desarrollo y la maduración presentaron en el momento de la cosecha una piel de color rojo intenso, con una relación $a / b$ de 0,45 , mientras que los frutos expuestos a la luz desarrollaron una coloración amarilla con una relación de color $a / b$ de 0,04 (Figura 1 y Tabla 1 ). El análisis de carotenoides en la piel de ambos frutos reveló que el contenido en carotenoides totales fue cuatro veces más elevado en la piel de los frutos rojos que en los amarillos, debido principalmente a que el contenido en el caroteno licopeno era cerca de 50 veces superior (Tabla 1).

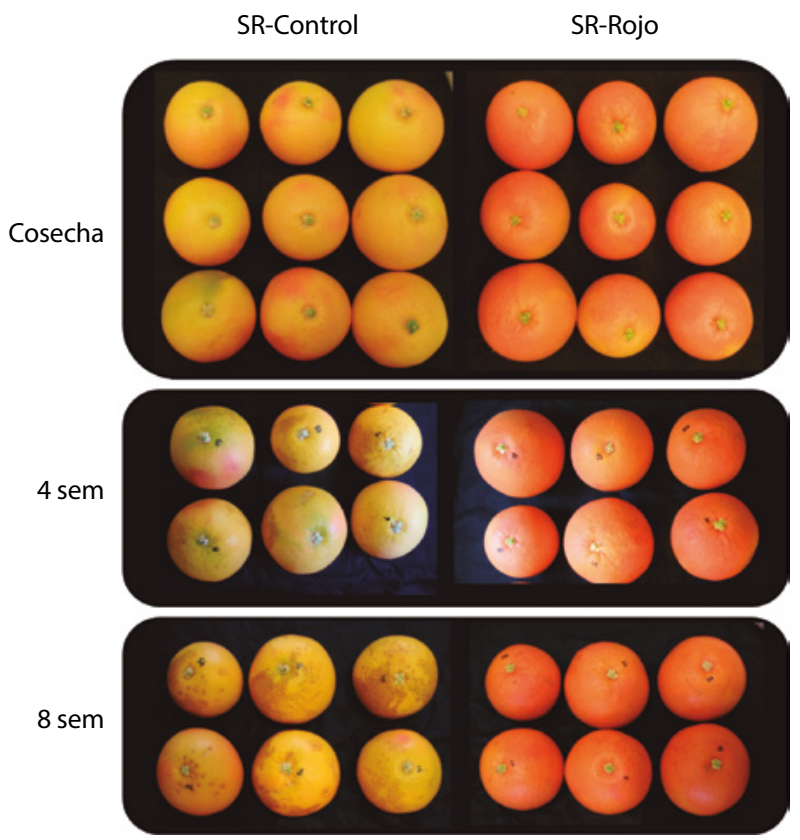

Figura 1. Síntomas de DF en la piel de frutos de pomelo SR control (SR-Control) y frutos rojos (SR-Rojo) en el momento de la cosecha y después de 4 y 8 semanas de almacenamiento a $2{ }^{\circ} \mathrm{C}$.

Los síntomas iniciales de DF se detectaron en aproximadamente un $7 \%$ de los frutos control tras dos semanas de conservación a $2{ }^{\circ} \mathrm{C}$ en frío. Conforme avanzó el periodo de conservación se incrementó la intensidad y el porcentaje de DF en los frutos de piel amarilla, alcanzando después de ocho semanas una incidencia severa que afectó al 93\% de los frutos (Tabla 1). En estas condiciones el aspecto visual de los frutos estuvo seriamente comprometido, con una elevada severidad de los síntomas que afectó a su aceptación comercial (Figura 1). En los frutos de color rojo, sin embargo, apenas se desarrollaron síntomas de DF, el cual alcanzó solamente a un 33\% de los frutos al finalizar el período de conservación y con una baja intensidad (0,5 en una escala de 0 a 3$)$. Estos frutos todavía mantienen su apariencia y calidad comercial a pesar de la presencia de pequeñas lesiones en la piel difícilmente detectables (Figura 1), y podrían superar los períodos cuarentenarios exigidos en las exportaciones.

\section{Evaluación de la capacidad antioxidante}

La evaluación de la capacidad del tejido para inactivar las especies reactivas del oxígeno, específicamente el oxígeno singlete (SOAC), mostró un valor entre dos a tres veces superior en los frutos rojos en el momento de la cosecha, que se mantuvo o incluso aumentó durante el período de conservación (Figura 2).

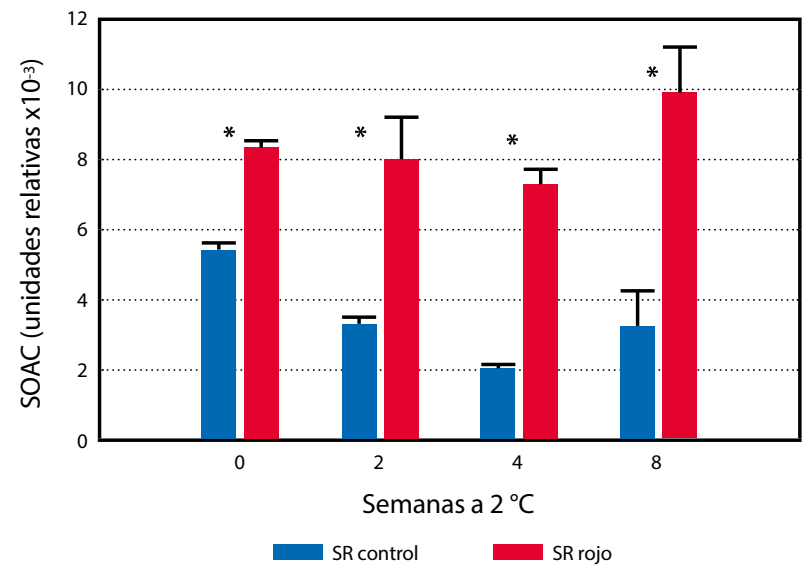

Figura 2. Capacidad para secuestrar el oxígeno singlete (SOAC-unidades relativas $\times 10^{-3}$ ) en la piel de frutos de pomelo SR control (SR-Control) y frutos rojos (SR-Rojo) en el momento de la cosecha y después de dos, cuatro y ocho semanas de almacenamiento a $2{ }^{\circ} \mathrm{C}$. Los asteriscos indican diferencias significativas entre frutos control y rojos (prueba $\mathrm{T}, \mathrm{p}<0,05)$.

\begin{tabular}{|c|c|c|c|c|c|c|}
\hline \multicolumn{3}{|c|}{} & \multicolumn{3}{|c|}{ Incidencia de DF (\%) } \\
\hline & $\begin{array}{c}\text { Color } \\
(\mathbf{a} / \mathrm{b})\end{array}$ & $\begin{array}{c}\text { Carotenoides } \\
(\mu \mathrm{g} / \mathrm{g} \text { PF) }\end{array}$ & $\begin{array}{c}\text { Licopeno } \\
(\mu \mathrm{g} / \mathrm{g} \text { PF) }\end{array}$ & 2 sem & 4 sem & 8 sem \\
\hline SR-control & $0,04 \pm 0,01 \mathrm{~b}$ & $20,9 \pm 0,80 \mathrm{~b}$ & $0,92 \pm 0,07 \mathrm{~b}$ & $6,7 \mathrm{a}$ & $73 \mathrm{a}$ & $93 \mathrm{a}$ \\
SR-rojo & $0,45 \pm 0,01 \mathrm{a}$ & $78,1 \pm 0,49 \mathrm{a}$ & $45,2 \pm 0,24 \mathrm{a}$ & $0,0 \mathrm{~b}$ & $13 \mathrm{~b}$ & $33 \mathrm{~b}$ \\
\hline
\end{tabular}

Tabla 1. Color de la piel, contenido de licopeno y de carotenoides totales en la piel de frutos de pomelos Star Ruby (frutos control y frutos rojos) en cosecha y porcentaje de frutos con daño por frío después de 2, 4 y 8 semanas de almacenamiento a $2{ }^{\circ} \mathrm{C}$. Medias seguidas de igual letra dentro de la misma columna no difieren significativamente (prueba $\mathrm{T}, \mathrm{p}<0,05$ ). 


\begin{tabular}{|c|c|c|c|c|}
\hline \multirow{2}{*}{} & \multicolumn{2}{|c|}{ GR } & \multicolumn{2}{c|}{ CAT } \\
\cline { 2 - 5 } & SRcontrol & SR-rojo & SRcontrol & SR-rojo \\
\hline Cosecha & $1,97 \pm 0,07$ & $1,64 \pm 0,11$ & $0,48 \pm 0,02 \mathrm{~b}$ & $2,47 \pm 0,23 \mathrm{a}$ \\
2 sem & $1,55 \pm 0,05$ & $1,65 \pm 0,12$ & $2,71 \pm 0,28 \mathrm{~b}$ & $4,62 \pm 0,23 \mathrm{a}$ \\
4 sem & $1,65 \pm 0,06$ & $1,61 \pm 0,07$ & $2,38 \pm 0,23 \mathrm{~b}$ & $4,76 \pm 0,28 \mathrm{a}$ \\
8 sem & $1,71 \pm 0,03$ & $1,79 \pm 0,07$ & $1,98 \pm 0,17 \mathrm{~b}$ & $4,05 \pm 0,40 \mathrm{a}$ \\
\hline \multirow{2}{*}{ Cosecha } & APX & & \multicolumn{2}{|c|}{ SOD } \\
\cline { 2 - 5 } 2 sem & $0,01 \pm 0$ & SR-rojo & $315,8 \pm 10,5 \mathrm{a}$ & $222,8 \pm 8,5 \mathrm{~b}$ \\
4 sem & $0,01 \pm 0$ & $0,01 \pm 0$ & $367,8 \pm 11,3$ & $399,5 \pm 7,0$ \\
\hline 8 sem & $0,01 \pm 0$ & $0,01 \pm 0$ & $279,1 \pm 5,6$ & $298,2 \pm 20,6$ \\
\hline
\end{tabular}

Tabla 2. Actividad (U /mg proteína) de las principales enzimas antioxidantes (catalasa-CAT, glutatión reductasa-GR, superóxido dismutasa-SOD y ascorbato peroxidasa-APX) en la piel de frutos de pomelo Star Ruby en el momento de la cosecha y después de dos, cuatro y ocho semanas de almacenamiento a $2{ }^{\circ} \mathrm{C}$. Medias seguidas de igual letra dentro de la misma enzima y fila no difieren significativamente, ausencia de letras significa ausencia de diferencias (prueba $\mathrm{T}, \mathrm{p}<0,05)$.

\begin{tabular}{|c|c|c|c|c|}
\hline & \multicolumn{2}{|c|}{ AsA (mg/100g PF) } & \multicolumn{2}{c|}{ GSH (nmol/g PF) } \\
\hline & SR-control & SR-rojo & SR-control & SR-rojo \\
\hline Cosecha & $307 \pm 9,2 \mathrm{a}$ & $122 \pm 3,4 \mathrm{~b}$ & $109 \pm 8,0$ & $125 \pm 8,0$ \\
2 sem & $217 \pm 6,5 \mathrm{a}$ & $115 \pm 1,3 \mathrm{~b}$ & $125 \pm 3,0$ & $110 \pm 6,0$ \\
$4 \mathrm{sem}$ & $227 \pm 4,2 \mathrm{a}$ & $109 \pm 9,1 \mathrm{~b}$ & $147 \pm 1,0$ & $156 \pm 2,0$ \\
$8 \mathrm{sem}$ & $211 \pm 3,3 \mathrm{a}$ & $102 \pm 5,2 \mathrm{~b}$ & $114 \pm 7,0 \mathrm{~b}$ & $141 \pm 4,0 \mathrm{a}$ \\
\hline
\end{tabular}

Tabla 3. Contenido de ácido ascórbico-AsA (mg/100g PF) y glutatión-GSH (nmol/g PF) en la piel de frutos de pomelo Star Ruby en el momento de la cosecha y después de dos, cuatro y ocho semanas de almacenamiento a $2{ }^{\circ} \mathrm{C}$. Medias seguidas de igual letra no difieren significativamente, ausencia de letras significa ausencia de diferencias (prueba $\mathrm{T}, \mathrm{p}<0,05$ ).

La actividad de las enzimas SOD, APX y GR no se modificó sustancialmente durante el almacenamiento en frío y tampoco se observaron diferencias entre los frutos control y los frutos rojos. Sin embargo, la actividad CAT fue superior en los frutos rojos desde el momento de la cosecha y permaneció más elevada durante todo el período de almacenamiento (Tabla 2). A su vez, la actividad de esta enzima aumentó por las bajas temperaturas, se incrementó a partir de la segunda semana de almacenamiento y permaneció elevada durante todo el periodo de conservación.

En relación al sistema antioxidante no enzimático, el contenido de AsA fue superior (x2) desde el momento de la cosecha en los frutos control, más sensibles a los DF, diferencia que se mantuvo durante todo el período de conservación (Tabla 3). A su vez, en el caso del glutatión los valores permanecieron relativamente constantes durante la conservación y sin diferencias entre los dos tipos de frutos evaluados (Tabla 3 ).

\section{Discusión}

Los frutos de pomelo son altamente sensibles a las bajas temperaturas de conservación y desarrollan DF a temperaturas inferiores a $10^{\circ} \mathrm{C}$. Sin embargo, los resultados de este trabajo demuestran que la presencia de cantidades importantes de licopeno en la piel minimizó la aparición de síntomas de DF durante al menos ocho semanas de conservación a $2{ }^{\circ} \mathrm{C}$ (Figura 1 y Tabla 1), sugiriendo que la presencia de este caroteno puede estar relacionada con la tolerancia a las bajas temperaturas. El licopeno es uno de los carotenoides con mayor capacidad antioxidante, cuya acción se ha relacionado con la inactivación de especies reactivas del oxígeno, principalmente el radical oxígeno singlete (Di Mascio, et al., 1989; Martínez y Melendez-Martínez, 2015). En este sentido, los daños por frío que se producen en los frutos cítricos durante la exposición a bajas temperaturas se han asociado a un proceso de estrés oxidativo, 
por lo que cobra sentido que este caroteno pueda vincularse a un aumento en la capacidad antioxidante del tejido resistente a desarrollar DF y, por tanto, a una mayor protección frente a las especies reactivas del oxígeno (Lado, et al., 2015a).

La capacidad para secuestrar el oxígeno singlete (SOAC) mostró un valor entre dos a tres veces superior en la piel de los frutos de pomelo rojo (Figura 2), en los que había aumentado más de 50 veces la concentración de este caroteno, lo que sugiere que esta especie reactiva de oxígeno podría potenciar el desarrollo de DF en los frutos de pomelo. Diferentes trabajos han demostrado que esta especie reactiva podría, además de provocar daño directo a nivel de las membranas celulares, actuar como una molécula señal en la activación del proceso de muerte celular programada (Apel y Hirt 2004; Wagner, et al., 2004) potenciando en este caso la aparición de los síntomas de daño. Es interesante destacar que la mayor capacidad SOAC en la piel de los frutos rojos ya se detectó en el momento de cosecha, lo que puede indicar que sería una característica inherente a la composición de la piel de estos frutos rojos. Alternativamente, también podría estar relacionado a que los frutos ya estuvieran expuestos en el campo a condiciones estresantes, posiblemente bajas temperaturas, que provocarían un aumento en estas especies tóxicas. El licopeno podría ejercer un importante papel en este proceso.

El sistema antioxidante de respuesta a diversos estreses en las plantas es muy complejo y se compone de un gran número de procesos que se activan, en este caso, en respuesta a las bajas temperaturas (Hossain, et al., 2012). Se destaca la activación de las enzimas antioxidantes CAT, SOD, APX, GR y otras (sistema enzimático) y los cambios en el contenido en los metabolitos antioxidantes como el AsA y el GSH (sistema no enzimático) (Sevillano, et al., 2009). En este sentido, se evaluó también la posible contribución de ambos sistemas en la protección frente al DF en los frutos de pomelos SR-control (sensibles) y SR-rojos (tolerantes). Entre las enzimas evaluadas, la única que incrementó su actividad en respuesta a las bajas temperaturas fue la CAT, mostrando también diferencias entre frutos sensibles y tolerantes al DF (Tabla 2). En el caso de los frutos cítricos se ha descrito que esta enzima es clave en la protección frente a las bajas temperaturas en frutos de diferentes variedades de mandarina (Sala y Lafuente, 1999; Lafuente, et al., 2004; Ghasemnezhad, et al., 2008). Sin embargo, el aumento de la actividad CAT en ambos tratamientos durante la exposición al frío (Tabla 2) sugiere que esta enzima estaría más relacionada con la respuesta a las bajas temperaturas y que los frutos rojos son capaces de mantener un nivel superior de actividad enzimática en estas condiciones. Por lo tanto, la mayor actividad CAT en frutos tolerantes podría estar contribuyendo solo parcialmente a la tolerancia al DF.

En relación a la actividad del resto de las enzimas del sistema antioxidante (APX, SOD y GR), los resultados indican que no parecen estar relacionadas con la tolerancia inducida al frío en frutos de pomelo, de forma similar a lo que se ha descrito en otros frutos sensibles al frío como el pepino (Qian, et al., 2013) o el mango (Chongchatuporn, et al., 2013). El estudio del sistema antioxidante no enzimático tampoco reveló una implicación con la tolerancia de los frutos de pomelo rojos a los DF, ya que los frutos control mostraron el doble de contenido de AsA que los frutos rojos durante todo el almacenamiento y no se encontraron diferencias en el contenido de glutatión (Tabla 3). Es así como estos metabolitos antioxidantes (AsA y GSH) pueden constituir más bien una respuesta de los frutos frente al estrés por frío no relacionada directamente con la protección o tolerancia frente a este estrés en los frutos de pomelo.

Es importante destacar entonces que, si bien existía alguna indicación en la literatura de que las zonas rojas del pomelo podrían mostrar una mayor tolerancia a los daños por frío, hasta la fecha no se había demostrado que la presencia del licopeno era responsable de esta tolerancia, lo que constituye una primera evidencia fisiológica de la relevancia del presente trabajo. En segundo lugar, se demuestra que no es a través del sistema antioxidante general, ni mediante otros antioxidantes como la vitamina $\mathrm{C}$ o el glutatión, sino que es el licopeno el compuesto antioxidante al que parece estar asociada esta tolerancia. En tercer lugar, se demuestra también que la presencia de licopeno en frutos potencia la capacidad del tejido para inactivar la especie reactiva oxígeno singlete en aquellos frutos más tolerantes, sugiriendo que esta especie reactiva parece estar mediando en el desarrollo de daños por frío en los cítricos. Finalmente, y desde una perspectiva aplicada, la recolección y clasificación de frutos de pomelo en base a la coloración roja de la piel es una posibilidad interesante de manejo para evitar los daños por frío y contribuir a superar las restricciones cuarentenarias. Es así como algunos países exportadores de cítricos ya están actualmente utilizando esta estrategia en los frutos que destinan a la exportación y requieren varias semanas de exposición a baja temperatura.

\section{Reconocimientos}

Nuestro agradecimiento a Amparo Beneyto por su excelente ayuda técnica. Este trabajo ha sido financiado por los proyectos (AGL2009-11558 y AGL2012-34573) del Ministerio de Economía y Competitividad. Al proyecto PROMETEOII/2014/027 (Generalitat Valenciana). Joanna Lado fue receptora de una beca JAE-Pre CSIC (Fondo Social Europeo) y forma parte del Instituto Nacional de Investigación Agropecuaria (INIA). Los autores de este trabajo pertenecen a la Red CYTED Ibercarot y Red de Excelencia CaRed BIO2015-71703-REDT (Ministerio de Economía y Competitividad, España).

\section{Referencias}

Aizawa, K., Iwasaki, Y., Ouchi, A., Inakuma. T., Nagaoka. S., Terao. J. y Mukai. K., 2011. Development of singlet oxygen absorption capacity (SOAC) assay method. 2. Measurements of the SOAC values for carotenoids and food extracts. En: Journal of Agricultural and Food Chemistry, 59, pp.3717-3729.

Alós, E., Rodrigo, MJ. y Zacarías, L., 2014. Differential transcriptional regulation of L-ascorbic acid content in peel and pulp of citrus fruits during development and maturation. En: Planta, 239, pp.1113-1128.

Alquezar, B., Rodrigo, MJ. y Zacarías, L., 2008. Regulation of carotenoid biosynthesis during fruit maturation in the redfleshed orange mutant Cara Cara. En: Phytochemistry, 69, pp.1997-2007.

Apel, K. y Hirt, H., 2004. Reactive oxygen species: metabolism, oxidative stress, and signal transduction. En: Annual Review of Plant Biology, 55, pp.373-399.

Arbona, V., Flors, V., Jacas, J., García-Agustín, P. y GómezCadenas, A., 2003. Enzymatic and non-enzymatic 
antioxidant responses of Carrizo citrange, a salt-sensitive citrus rootstock, to different levels of salinity. En: Plant \& Cell Physiology, 44, pp.388-394.

Bradford, MM., 1976. A rapid and sensitive method for the quantitation of microgram quantities of protein utilizing the principle of protein-dye binding. En: Analytical Biochemistry, 72, pp.248-254.

Chongchatuporn, U., Ketsa, S., van Doorn, WG., 2013. Chilling injury in mango (Mangifera indica) fruit peel: Relationship with ascorbic acid concentrations and antioxidant enzyme activities. En: Postharvest Biology and Technology, 86, pp.409-417.

Ghasemnezhad, M., Marsh, K., Shilton, R., Babalar, M. y Woolf, A., 2008. Effect of hot water treatments on chilling injury and heat damage in "satsuma" mandarins : Antioxidant enzymes and vacuolar ATPase, and pyrophosphatase. En: Postharvest Biology and Technology, 48, pp.364-371.

Hossain, Z., Nouri, MZ. y Komatsu, S., 2012. Plant cell organelle proteomics in response to abiotic stress. En: Journal of Proteome Research, 11, pp.37-48.

Kim, SH., Ahn, YO., Ahn, MJ., Lee, H-S. y Kwak, SS., 2012. Down-regulation of $\beta$-carotene hydroxylase increases $\beta$-carotene and total carotenoids enhancing salt stress tolerance in transgenic cultured cells of sweetpotato. En: Phytochemistry, 74, pp.69-78.

Lado, J., Rodrigo, MJ., Cronje, P. y Zacarías, L., 2015a. Involvement of lycopene in the induction of tolerance to chilling injury in grapefruit. En: Postharvest Biology and Technology, 100, pp.176-186.

Lado, J., Cronje, P., Alquézar, B., Page, A., Manzi, M., GómezCadenas, A., Stead, AD., Zacarías, L. y Rodrigo, MJ., $2015 \mathrm{~b}$. Fruit shading enhances peel color, carotenes accumulation and chromoplast differentiation in red grapefruit. En: Physiologia Plantarum, 154, pp.469-484.

Lafuente, MT., Martínez-Téllez, MA. y Zacarías, L., 1997. Abscisic acid in the response of "fortune " mandarins to chilling. Effect of maturit y and high-temperature conditioning. En: Journal of the Science of Food and Agriculture, 73, pp.494-502.

Lafuente, MT., Sala, JM. y Zacarias, L., 2004. Active oxygen detoxifying enzymes and phenylalanine ammonia-lyase in the ethylene-induced chilling tolerance in citrus fruit. En: Journal of Agricultural and Food Chemistry, 52, pp.3606-3611.

Lafuente, MT. y Zacarías, L., 2006. Postharvest physiological disorders in citrus fruit. En: Stewart Postharvest Review, 2, pp.1-9.

Martínez, A., Melendez-Martínez, AJ., 2015. Lycopene, oxidative cleavage derivatives and antiradical activity. En:
Computational and Theoretical Chemistry, 1077, pp.92-98.

Di Mascio, P., Kaiser, S. y Sies, H., 1989. Lycopene as the most efficient biological carotenoid singlet oxygen quencher. En: Archives of Biochemistry and Biophysics, 274, pp.532-538.

Ouchi, A., Aizawa, K., Iwasaki, Y., Inakuma, T., Terao, J., Nagaoka, S. y Mukai, K., 2010. Kinetic study of the quenching reaction of singlet oxygen by carotenoids and food extracts in solution. Development of a singlet oxygen absorption capacity (SOAC) assay method. En: Journal of Agricultural and Food Chemistry, 58, pp.9967-9978.

Qian, C., He, Z., Zhao, Y., Mi, H., Chen, X. y Mao, L., 2013. Maturity-dependent chilling tolerance regulated by the antioxidative capacity in postharvest cucumber (Cucumis sativus L.) fruits. En: Journal of the Science of Food and Agriculture, 93, pp.626-633.

Rodrigo, MJ., Marcos, J., Alférez, F., Mallent, D. y Zacarías, L., 2003. Characterization of Pinalate, a novel Citrus sinensis mutant with a fruit-specific alteration that results in yellow pigmentation and decreased ABA content. En: Journal of Experimental Botany, 54, pp.727-738.

Sala, JM. y Lafuente, MT., 1999. Catalase in the heat-induced chilling tolerance of cold-stored hybrid Fortune mandarin fruits. En: Journal of Agricultural and Food Chemistry, 47, pp.2410-2414.

Sala, JM. y Lafuente, MT., 2000. Catalase enzyme activity is related to tolerance of mandarin fruits to chilling. En: Postharvest Biology and Technology, 20, pp.81-89.

Sevillano, L., Sanchez-Ballesta, MT., Romojaro, F. y Flores, FB., 2009. Physiological, hormonal and molecular mechanisms regulating chilling injury in horticultural species. Postharvest technologies applied to reduce its impact. En: Journal of the Science of Food and Agriculture, 89, pp.555-573.

Singh, P. yGoyal, GK., 2008. Lycopene : its properties and anticarcinogenic effects. En: Comprehensive Reviews in Food Science and Food Safety, 7, pp.255-270.

Toivonen, PMA., 2004. Postharvest storage procedures and oxidative stress. En: Hortscience, 39, pp.938-942.

Wagner, D., Przybyla, D., Op den Camp, R., Kim, C., Landgraf, F., Lee, KP., Würsch, M., Laloi, C., Nater, M., Hideg, E. y Apel, K., 2004. The genetic basis of singlet oxygen-induced stress responses of Arabidopsis thaliana. En: Science, 306, pp.1183-1185.

Zhang, Y., Butelli, E., De Stefano, R., Schoonbeek, H-J., Magusin, A., Pagliarani, C., Wellner, N., Hill, L., Orzaez, D., Granell, A., Jones, JDG. y Martin. C., 2013. Anthocyanins double the shelf life of tomatoes by delaying overripening and reducing susceptibility to gray mold. En: Current Biology, 23, pp.1094-1100. 